Supplementary Information for

\title{
Globalization-driven industry relocation significantly reduces Arctic
}

\section{PAH contamination}

\section{Authors:}

Lulu Lian ${ }^{1}$, Tao Huang ${ }^{1 *}$, Xianmin $\mathrm{Ke}^{3,4}$, Zaili Ling ${ }^{5}$, Wanyanhan Jiang ${ }^{6}$, Zhanxiang Wang ${ }^{1}$, Shijie Song ${ }^{1}$, Jixiang $\mathrm{Li}^{2}$, Yuan Zhao ${ }^{1}$, Hong Gao ${ }^{1}$, Shu Tao ${ }^{2}$, Junfeng $\mathrm{Liu}^{2}$, Jianmin $\mathrm{Ma}^{2,1 *}$

\section{Affiliations:}

${ }^{1}$ Key Laboratory for Environmental Pollution Prediction and Control, Gansu Province, College of Earth and Environmental Sciences, Lanzhou University, Lanzhou, 730000, P. R. China

${ }^{2}$ Laboratory for Earth Surface Processes, College of Urban and Environmental Sciences, Peking University, Beijing, 100871, P. R. China

${ }^{3}$ School of Water and Environment, Chang'an University, Xi'an, 710054, Shaanxi, P. R. China

${ }^{4}$ Key Laboratory of Subsurface Hydrology and Ecological Effect in Arid Region of the Ministry of Education, Chang'an University, Xi' an, 710054, Shaanxi, P. R. China ${ }^{5}$ College of Agricultural and Forestry Economics \& Management, Lanzhou University of Finance and Economics, Lanzhou 730000, P. R. China

${ }^{6}$ School of Public Health, Chengdu University of Traditional Chinese Medicine, Chengdu, 610000, P. R. China

Correspondence: Tao Huang (huangt@1zu.edu.cn); Jianmin Ma (jmma@pku.edu.cn) 
This Supporting Information includes 3 sections of text, 5 Supporting Tables, and 13 Supporting Figures.

\section{Contents}

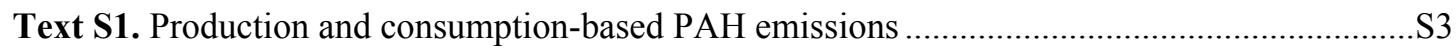

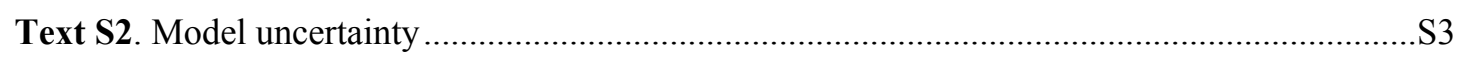

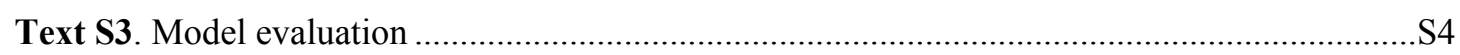

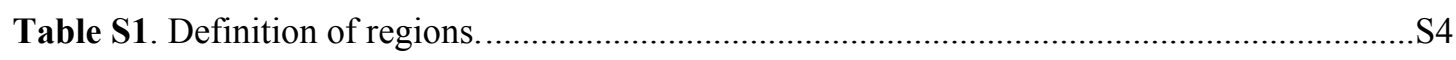

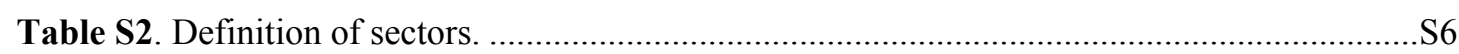

Table S3. Definition of three model scenarios performed in this study. ........................................ 7

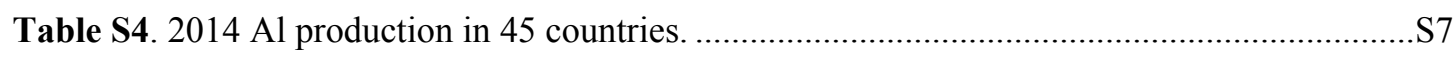

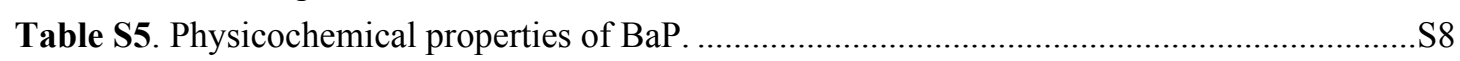

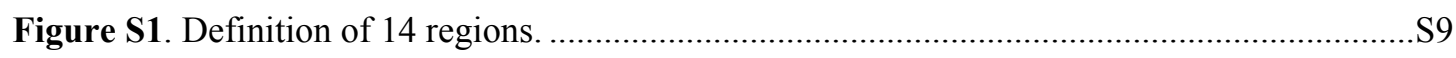

Figure S2. Comparison between modeled and measured $\mathrm{BaP}$ air concentrations..........................S9

Figure S3. BaP emission and emission fraction in 2014 ......................................................S10

Figure S4. BaP emissions from all sources and industrial sources in 2014, collected from the PKU-

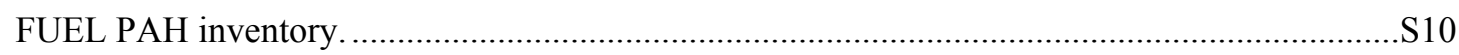

Figure S5. Per capital PAHs and BaP emissions across the global under TRADE scenario in $2014 .$.

Figure S6. Comparison between PAH production-based and consumption-based emissions in 14 regions in 2014.

Figure S7. Comparison between BaP production-based and consumption-based emissions in 14 regions in 2014.

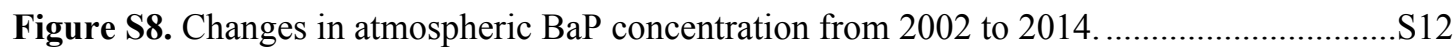

Figure S9. Changes in BaP annual emissions from 2002 to 2014 ............................................. 13

Figure S10. Net international BaP emission flows and interregional BaP emission flows within globe in 2014.

Figure S11. CanMETOP simulated annually averaged BaP concentration across the globe from TRADE run and NO_TRADE run in 2014, the fraction of BaP concentration fraction of TRADE to NO_TRADE run. S14

Figure S12. Relative contributions from different regions to the BaP concentration over the Arctic from TRADE scenario and NO_TRADE scenario.

Figure S13. BaP dry and wet deposition fluxes across the global under TRADE scenario in 2014. 


\section{Text S1. Production and consumption-based PAH emissions}

The production-based PAH emissions for each sector and region can be estimated as follows:

$$
E_{a, i}=E A_{a, i} \times \sum_{f}\left(E F_{a, i, f} \times A_{a, i, f}\right),
$$

where $a, i$, and $f$ represent the region, sector, and technology mode for combustion and industrial processes, respectively; $E A$ denotes fuel consumption; $A$ denotes the fraction of fuel or production consumed by a specific form of technology in a given sector; and $E F$ is the emission factor.

Based on production and pollution control technologies in different sectors, Shen et al. compile the latest and most comprehensive estimates of regional emission factors and associated speciation profiles for all anthropogenic PAH sources. ${ }^{1}$ The emission factors used in this study and technology distributions were obtained from Shen et al. ${ }^{1}$

We obtained production-based PAH emissions for each sector and region by multiplying multiply emission factors and speciation profiles by energy usage or product yield.

In traditional block form, the normalized MRIO is written as follows:

$$
\left(\begin{array}{c}
x_{1} \\
x_{2} \\
\vdots \\
x_{m}
\end{array}\right)=\left(\begin{array}{cccc}
A_{11} & A_{12} & \cdots & A_{1 m} \\
A_{21} & A_{22} & \cdots & A_{2 m} \\
\vdots & \vdots & \ddots & \vdots \\
A_{m 1} & A_{m 2} & \cdots & A_{m m}
\end{array}\right)\left(\begin{array}{c}
x_{1} \\
x_{2} \\
\vdots
\end{array}\right)+\sum_{b}\left(\begin{array}{c}
y_{1 b} \\
y_{2 b} \\
\vdots \\
y_{m b}
\end{array}\right),
$$

where $\boldsymbol{x}_{\boldsymbol{a}}$ is a vector of the total economic monetary output of each sector in region $\boldsymbol{a} ; \boldsymbol{y}_{\boldsymbol{a}} \boldsymbol{b}$ is a vector of the finished demand (investment, government consumption, and household consumption) by each sector produced in region $\boldsymbol{a}$ and consumed in region $\boldsymbol{b}$; and $\boldsymbol{A}_{\boldsymbol{a} \boldsymbol{b}}$ is a normalized matrix of intermediate requirements from region $\boldsymbol{a}$ to region $\boldsymbol{b}$. When solving from total output, Eq. S2 can be rewritten as follows:

$$
X=(I-A)^{-1} Y,
$$

where $(\boldsymbol{I}-\boldsymbol{A})^{-\mathbf{1}}$ is the Leontief inverse matrix.

Based on the PAH production-based emission inventory, we estimate region- and sectorspecific consumption-based PAH emissions by incorporating Eq. S3. Consumption-based PAH emissions can then be derived by the following relationship:

$$
\boldsymbol{E}_{\boldsymbol{c}}=\widehat{\boldsymbol{E} I}\left[(\boldsymbol{I}-\boldsymbol{A})^{-1} \boldsymbol{Y}\right],
$$

where $\boldsymbol{E}_{\boldsymbol{c}}$ denotes region- and sector-specific consumption-based PAH emissions; $\boldsymbol{E} \boldsymbol{I}$ denotes emission intensity for 39 sectors in 141 GTAP regions; and $\widehat{\boldsymbol{E} I}$ is the diagonalization of $\boldsymbol{E} \boldsymbol{I}$. Regionand sector-specific emission intensity $(\boldsymbol{E} \boldsymbol{I})$ is obtained as sectoral production-based emissions (described in section 2.2) divided by sectoral outputs for each region.

Moreover, a comparison of different global MRIO databases showed that PAH emissions embodied in international trade vary by up to $13 \%$, and the observed differences among the MRIO results were close to the differences in the underlying production-based inventories, indicating that the MRIO-related error is smaller than the error produced in a production-based emission inventory. ${ }^{2}$

\section{Text S2. Model uncertainty}

Monte Carlo analysis always used to quantify uncertainties the uncertainties of the transport 
model, exposure, and risk analysis ${ }^{3}$, which requires parameterization of the degree of uncertainty and the shape of input distributions associated with each input parameter. Here, the global CanMETOP model includes complex dynamical and physical processes, including deposition and precipitation scavenging, horizontal and vertical advection, turbulent diffusion, and exchange between environmental media. As such, this study used a first-order error propagation approach to calculate the uncertainties in modeled concentrations ${ }^{4}\left(C f_{O}\right)$. Uncertainties were calculated using the formula

$$
C f_{o}=\exp \sqrt{\sum_{i}\left(\ln C f_{i}\right)^{2} \times S_{i}^{2}},
$$

where $C f_{o}$ and $C f_{i}$ identify the confidence factors that span the $95 \%$ confidence interval around the median of a log-normally distributed variable, respectively; $S_{i}$ is the relative sensitivity of the model output subject to changes in input parameter $i$, of which $S$ is calculated by

$$
S=\left({ }^{\Delta O} / O\right) /\left({ }^{\Delta I} / I\right)
$$

where $\Delta O$ and $\Delta I$ are the relative changes in input $(I)$ and output $(O)$ parameters of interest, respectively.

In this study, we only considered the parameters significantly affecting PAH emissions and modeling results. For the PAH emissions, variation intervals were set to be $10 \%$ for all sources. ${ }^{1}$ For the CanMETOP model, the uncertainties of physicochemical properties of $\mathrm{BaP}$, $\mathrm{Cf}$ values are listed in Table S5. Finally, we estimate an overall uncertainty of global production-based PAH emissions with factors of 4.86, and BaP air concentrations over Arctic with factors of 1.76 in 2014, respectively.

\section{Text S3. Model evaluation}

The CanMETOP model has been extensively evaluated and validated. ${ }^{4-6}$ Here, the model was run from 2002 to 2014 to build up the equilibrium status of PAH concentrations in the surface via comparison of measured data with modeled concentrations. The results as shown in Fig S12. The statistics between modeled and sampled BaP air concentrations at 196 sites in the globe, collected from research group and literatures. We used several international monitoring programs for POPs have been established since the 1990s under the umbralla of EMEP (Co-operative Programme for Monitoring and Evaluation of the long-range transmissions of air pollutants in Europe), IADN (Integrated Atmospheric Deposition Network), AMAP (Arctic Monitoring and Assessment Programme). The modeled concentrations agree well with the measured data at $r=0.629(p<0.01)$. The errors with two orders of magnitude between modeled and sampled results could be resulted from a spatial mismatch between sampling sites and the model grid with the area of $1^{\circ} \times 1^{\circ}$ latitude/longitude.

Table S1. Definition of regions.

\begin{tabular}{clll}
\hline $\begin{array}{c}\text { Num } \\
\text { ber }\end{array}$ & \multicolumn{1}{c}{ Description } & \multicolumn{1}{c}{ 141 GTAP_name } & \multicolumn{1}{c}{ 207 Country_name } \\
\hline 1 & China & China, Hong Kong, Taiwan & $\begin{array}{l}\text { China, Hong Kong, Taiwan, } \\
\text { Macau }\end{array}$ \\
\hline 2 & Rest of East Asia & $\begin{array}{l}\text { Japan, Korea, Mongolia, Rest } \\
\text { of East Asia }\end{array}$ & $\begin{array}{l}\text { Japan, Korea, Democratic } \\
\text { People's Republic of Korea }\end{array}$ \\
\hline 3 & India & India & India \\
\hline
\end{tabular}




\begin{tabular}{|c|c|c|c|}
\hline 4 & $\begin{array}{l}\text { Central and } \\
\text { Northern Asia }\end{array}$ & $\begin{array}{l}\text { Mongolia, Pakistan, } \\
\text { Kazakhstan, Kyrgyzstan, } \\
\text { Tajikistan, Rest of Former } \\
\text { Soviet }\end{array}$ & $\begin{array}{lr}\text { Mongolia, } & \text { Pakistan, } \\
\text { Kazakhstan, } & \text { Kyrgyzstan, } \\
\text { Tajikistan, } & \text { Afghanistan, } \\
\text { Turkmenistan, Uzbekistan }\end{array}$ \\
\hline 5 & Southeast Asia & $\begin{array}{l}\text { Cambodia, Indonesia, Lao } \\
\text { People's Democratic Republic, } \\
\text { Malaysia, Philippines, } \\
\text { Singapore, Thailand, Viet } \\
\text { Nam, Brunei Darussalam, } \\
\text { Myanmar, Rest of Southeast } \\
\text { Asia, Bangladesh, Nepal, Sri } \\
\text { Lanka, Rest of South Asia }\end{array}$ & $\begin{array}{l}\text { Cambodia, Indonesia, Lao } \\
\text { People's Democratic Republic, } \\
\text { Malaysia, Philippines, } \\
\text { Singapore, Thailand, Viet } \\
\text { Nam, Brunei Darussalam, } \\
\text { Myanmar, Timor-Leste, } \\
\text { Bangladesh, Nepal, Sri Lanka, } \\
\text { Bhutan, the Maldives }\end{array}$ \\
\hline 6 & Russia & Russian Federation & Russian Federation \\
\hline 7 & Western Europe & $\begin{array}{l}\text { Austria, Belgium, Cyprus, } \\
\text { Czech Republic, Denmark, } \\
\text { Finland, France, Germany, } \\
\text { Greece, Ireland, Italy, } \\
\text { Luxembourg, Malta, } \\
\text { Netherlands, Portugal, Spain, } \\
\text { Sweden, United Kingdom, } \\
\text { Switzerland, Norway, Rest of } \\
\text { European FTA }\end{array}$ & $\begin{array}{l}\text { Austria, Belgium, } \\
\text { Czech Republic, Denmark, } \\
\text { Finland, France, Guadeloupe, } \\
\text { Germany, Greece, Ireland, } \\
\text { Italy, Luxembourg, Malta, } \\
\text { Netherlands, Bonaire, } \\
\text { Portugal, Spain, Sweden, } \\
\text { United Kingdom, Switzerland, } \\
\text { Norway, Iceland }\end{array}$ \\
\hline 8 & Eastern Europe & $\begin{array}{l}\text { Estonia, Hungary, Latvia, } \\
\text { Lithuania, Poland, Slovakia, } \\
\text { Slovenia, Albania, Bulgaria, } \\
\text { Belarus, Croatia, Romania, } \\
\text { Ukraine, Rest of Eastern } \\
\text { Europe, Rest of Europe }\end{array}$ & $\begin{array}{l}\text { Estonia, Hungary, Latvia, } \\
\text { Lithuania, Poland, Slovakia, } \\
\text { Slovenia, Albania, Bulgaria, } \\
\text { Belarus, Croatia, Romania, } \\
\text { Ukraine, Moldova, Kosovo, } \\
\text { Bosnia and Herzegovina, } \\
\text { Former Yugoslav Republic of } \\
\text { Macedonia, Montenegro, } \\
\text { Serbia, Gibraltar }\end{array}$ \\
\hline 9 & S. & ica & United States of America \\
\hline 10 & da & anada & Canada \\
\hline 11 & $\begin{array}{l}\text { Middle East and } \\
\text { North Africa }\end{array}$ & $\begin{array}{l}\text { Armenia, Azerbaijan, Georgia, } \\
\text { Bahrain, Islamic Republic of } \\
\text { Iran, Israel, Kuwait, Oman, } \\
\text { Qatar, Saudi Arabia, Turkey, } \\
\text { United Arab Emirates, Jordan, } \\
\text { Rest of Western Asia, Egypt, } \\
\text { Morocco, Tunisia, Rest of } \\
\text { North Africa }\end{array}$ & $\begin{array}{l}\text { Armenia, Azerbaijan, Georgia, } \\
\text { Bahrain, Islamic Republic of } \\
\text { Iran, Israel, Kuwait, Oman, } \\
\text { Qatar, Saudi Arabia, Turkey, } \\
\text { United Arab Emirates, Jordan, } \\
\text { Iraq, Lebanon, Syrian Arab } \\
\text { Republic, Yemen, Egypt, } \\
\text { Morocco, Tunisia, Algeria, } \\
\text { Libya }\end{array}$ \\
\hline 12 & Latin America & $\begin{array}{l}\text { Costa Rica, Guatemala, } \\
\text { Honduras, Nicaragua, Panama, } \\
\text { EI Salvador, Rest of Central } \\
\text { America, Dominican Republic, } \\
\text { Jamaica, Puerto Rico, Trinidad } \\
\text { and Tobago, Caribbean, } \\
\text { Argentina, Bolivia, Brazil, } \\
\text { Chile, Colombia, Ecuador, } \\
\text { Paraguay, Peru, Uruguay, } \\
\text { Venezuela, Rest of South } \\
\text { America, Mexico }\end{array}$ & $\begin{array}{l}\text { Costa Rica, Guatemala, } \\
\text { Honduras, Nicaragua, Panama, } \\
\text { EI Salvador, Belize, } \\
\text { Dominican Republic, Jamaica, } \\
\text { Puerto Rico, Trinidad and } \\
\text { Tobago, Antigua and Barbuda, } \\
\text { Aruba, the Bahamas, } \\
\text { Barbados, the British Virgin } \\
\text { Islands, the Cayman Islands, } \\
\text { Cuba, Grenada, Haiti, } \\
\text { Montserrat, Saint Kitts and } \\
\text { Nevis, Saint Lucia, Saint } \\
\text { Vincent and the Grenadines, } \\
\text { the Turks and Caicos Islands, }\end{array}$ \\
\hline
\end{tabular}




\begin{tabular}{|c|c|c|c|}
\hline & & & $\begin{array}{l}\text { Curaçao, Sint } \\
\text { Argentina, Bolivia, Brazilen, } \\
\text { Chile, Colombia, Ecuador, } \\
\text { Paraguay, Peru, Uruguay, } \\
\text { Venezuela, the Falkland } \\
\text { Islands (Malvinas), French } \\
\text { Guiana, Guyana, Suriname, } \\
\text { Mexico }\end{array}$ \\
\hline 13 & $\begin{array}{l}\text { Sub-Saharan } \\
\text { Africa }\end{array}$ & $\begin{array}{l}\text { Cameroon, Cote d'lvoire, } \\
\text { Ghana, Guinea, Nigeria, } \\
\text { Senegal, Benin, Burkina Faso, } \\
\text { Togo, Rest of Western Africa, } \\
\text { Central Africa, South Central } \\
\text { Africa, Ethiopia, Kenya, } \\
\text { Madagascar, Malawi, } \\
\text { Mauritius, Mozambique, } \\
\text { Tanzania, Uganda, Zimbabwe, } \\
\text { Rwanda, Rest of Eastern } \\
\text { Africa, Botswana, Namibia, } \\
\text { South Africa, Rest of South } \\
\text { African }\end{array}$ & $\begin{array}{l}\text { Cameroon, Cote } \\
\text { Ghana, Guinea, Nire, } \\
\text { Senegal, Benin, Burkina Faso, } \\
\text { Togo, Cabo Verde, Guinea- } \\
\text { Bissau, Liberia, } \\
\text { Mauritania, Niger, Sierra } \\
\text { Leone, Gambia, Central } \\
\text { African Republic, Chad, } \\
\text { Congo, Equatorial Guinea, } \\
\text { Gabon, Sao Tome and } \\
\text { Principe, Angola, Democratic } \\
\text { Republic of the Congo, } \\
\text { Ethiopia, Kenya, Madagascar, } \\
\text { Malawi, Mauritius, } \\
\text { Mozambique, Tanzania, } \\
\text { Uganda, Zimbabwe, Rwanda, } \\
\text { Sudan, Burundi, Comoros, } \\
\text { Djibouti, Eritrea, the } \\
\text { Seychelles, Somalia, South } \\
\text { Sudan, Botswana, Namibia, } \\
\text { South Africa, Lesotho, } \\
\text { Swaziland }\end{array}$ \\
\hline 14 & Rest of the World & $\begin{array}{l}\text { Australia, New Zealand, Rest } \\
\text { of Oceania, Rest of North } \\
\text { America, Rest of the World }\end{array}$ & $\begin{array}{l}\text { Australia, New Zealand, Cook } \\
\text { Islands, Fiji, French Polynesia, } \\
\text { Kiribati, New Caledonia, } \\
\text { Palau, Papua New Guinea, } \\
\text { Samoa, the Solomon Islands, } \\
\text { Tonga, Vanuatu, Bermuda, } \\
\text { Saint Pierre and Miquelon }\end{array}$ \\
\hline
\end{tabular}

Table S2. Definition of sectors.

\begin{tabular}{|c|c|c|c|}
\hline Num. & Sector & Sub-sector & GTAP-sector \\
\hline 1 & Residential & Residential & \\
\hline 2 & $\begin{array}{l}\text { Agriculture, } \\
\text { fishing }\end{array}$ & Agriculture/forestry; Fishing & $\begin{array}{l}\text { Paddy rice; Wheat; Cereal } \\
\text { grains nec*; Vegetables, fruit, } \\
\text { nuts; Oil seeds; Sugar cane, } \\
\text { sugar beet; Plant-based fibers; } \\
\text { Crops nec*; Bovine cattle, } \\
\text { sheep and goats, horses; Animal } \\
\text { products nec*; Raw milk; Wool, } \\
\text { silk-worm cocoons; Forestry; } \\
\text { Fishing }\end{array}$ \\
\hline 3 & $\begin{array}{l}\text { Coke, petroleum } \\
\text { products }\end{array}$ & $\begin{array}{lll}\begin{array}{l}\text { Coke } \\
\text { plants }\end{array} & \text { plants; } & \text { Petrochemical } \\
\end{array}$ & Petroleum, coal products \\
\hline 4 & Energy production & $\begin{array}{l}\text { Electricity plants; CHP plants; } \\
\text { Heat plants; Gas works; Oil } \\
\text { refineries; Liquefaction } \\
\text { plants; Other transformation; }\end{array}$ & $\begin{array}{l}\text { Coal; Oil; Gas; Electricity; Gas } \\
\text { manufacture, distribution; } \\
\text { Water }\end{array}$ \\
\hline
\end{tabular}




\begin{tabular}{|c|c|c|c|}
\hline & & $\begin{array}{l}\text { Fuel mining and extraction; } \\
\text { Oil refineries; Elec., CHP and } \\
\text { heat plants; Pumped storage } \\
\text { plants; Other energy ind. own } \\
\text { use }\end{array}$ & \\
\hline 5 & Industry & $\begin{array}{l}\text { Blast furnaces; Iron and steel; } \\
\text { Chemical and petrochemical; } \\
\text { Non-ferrous metals; Non- } \\
\text { metallic minerals; Transport } \\
\text { equipment; Machinery; Mining } \\
\text { and quarrying; Al Production; } \\
\text { Food and tobacco; Paper, pulp } \\
\text { and print; Wood and wood } \\
\text { products; Textile and leather; } \\
\text { Non-specified }\end{array}$ & $\begin{array}{l}\text { Other Extraction (formerly } \\
\text { Other minerals nec*, Minerals } \\
\text { nec*); Bovine meat products; } \\
\text { Meat products nec*; Vegetable } \\
\text { oils and fats; Dairy products; } \\
\text { Processed rice; Sugar; Food } \\
\text { products nec*; Beverages and } \\
\text { tobacco products; Textiles; } \\
\text { Wearing apparel; Leather } \\
\text { products; Wood products; Paper } \\
\text { products, publishing; Chemical } \\
\text { products; Basic pharmaceutical } \\
\text { products; Rubber and plastic } \\
\text { products; Mineral products } \\
\text { nec*; Ferrous metals; Metals } \\
\text { nec*; Metal products; } \\
\text { Computer, electronic and } \\
\text { optical products; Electrical } \\
\text { equipment; Machinery and } \\
\text { equipment nec*; Motor vehicles } \\
\text { and parts; Transport equipment } \\
\text { nec*; Manufactures nec* }\end{array}$ \\
\hline 6 & Transportation & $\begin{array}{l}\text { Domestic aviation; Road; Rail; } \\
\text { Pipeline transport; Domestic } \\
\text { navigation }\end{array}$ & $\begin{array}{l}\text { Transport nec*; } \\
\text { transport; Air transport }\end{array}$ \\
\hline 7 & Construction & Construction & Construction \\
\hline 8 & Other services & $\begin{array}{l}\text { Comm. and public services; } \\
\text { Other services }\end{array}$ & $\begin{array}{l}\text { Trade; Accommodation, Food } \\
\text { and service activities; } \\
\text { Warehousing and support } \\
\text { activities; Communication; } \\
\text { Financial services nec*; } \\
\text { Insurance (formerly Insurance); } \\
\text { Real estate activities; Business } \\
\text { services nec*; Recreational and } \\
\text { other services; Public } \\
\text { Administration and defense; } \\
\text { Education; Human health and } \\
\text { social work activities; } \\
\text { Dwellings }\end{array}$ \\
\hline
\end{tabular}

*nec is the abbreviate of "not elsewhere classified".

Table S3. Definition of three model scenarios performed in this study.

\begin{tabular}{lll}
\hline Scenario & Definition & Emission \\
\hline TRADE & $\begin{array}{l}\text { Production-based emissions from } \\
\text { PKU-BaP inventory }\end{array}$ & $E_{T R A D E}$ \\
NO_TRADE & $\begin{array}{l}\text { Eliminate emissions embodied in } \\
\text { interregional trade for each sector } \\
\text { of all regions in TRADE scenario }\end{array}$ & $E_{\text {NO_TRADE }}=E_{\text {TRADE }} \times\left(1-\frac{E_{\text {ex }}-E_{\text {im }}}{E_{T R A D E}}\right)$ \\
BASE & $\begin{array}{l}\text { Including all emission sources } \\
\text { from PKU-BaP inventory }\end{array}$ & $E_{B A S E}$ \\
\hline
\end{tabular}


Table S4. 2014 Al production (unit: Thousand metric tons) in 45 countries.

\begin{tabular}{llllll}
\hline \multicolumn{1}{c}{ Country } & Al production & Country & Al production & Country & Al production \\
\hline Australia & 1704 & Turkey & 54 & Kazakhstan & 208 \\
\hline Canada & 2858 & $\begin{array}{l}\text { United } \\
\text { Kingdom }\end{array}$ & 42 & Malaysia & 400 \\
\hline France & 360 & $\begin{array}{l}\text { United } \\
\text { States }\end{array}$ & 1710 & Montenegro & 44 \\
\hline Germany & 531 & Argentina & 443 & $\begin{array}{l}\text { Mozambiqu } \\
\text { e }\end{array}$ & 567 \\
\hline Greece & 173 & Azerbaijan & 50 & Nigeria & 0 \\
\hline Iceland & 800 & Bahrain & 931 & Oman & 364 \\
\hline Italy & 0 & Bosnia \& & 100 & Qatar & 640 \\
\hline Japan & 46 & Herzegovina & & Romania & 263 \\
\hline $\begin{array}{l}\text { Netherlan } \\
\text { ds }\end{array}$ & 0 & Brazil & 962 & Russia & 3300 \\
\hline $\begin{array}{l}\text { New } \\
\text { Zealand }\end{array}$ & 328 & Cameroon & 93 & Saudi & 665 \\
\hline Norway & 1250 & China & 28300 & Arabia & \\
\hline Slovakia & 168 & Egypt & 304 & South & 745 \\
\hline Slovenia & 85 & Africa & \\
\hline Spain & 230 & India & 38 & Tajikistan & 121 \\
& & Indonesia & 211 & Ukraine & 0 \\
\hline Sweden & 113 & United & 2330 \\
\hline & & Arab & Emirates \\
\hline
\end{tabular}

Table S5. Physicochemical properties of BaP used in the present study ${ }^{7}$.

\begin{tabular}{lll}
\hline Parameters & Value & $C f$ \\
\hline Molecular mass $(\mathrm{g} / \mathrm{mol})$ & 252.3 & 1 \\
Molar volume $\left(\mathrm{cm}^{3} / \mathrm{mol}\right)$ & 222.8 & 1 \\
Melting point $\left({ }^{\circ} \mathrm{C}\right)$ & 181.1 & 1 \\
Entropy of Fusion $(\mathrm{J} / \mathrm{mol} \mathrm{K})$ & 38.5 & 1 \\
Half-Lives in air $(\mathrm{h})$ & 5.3 & 2 \\
Half-Lives in soil $(\mathrm{d})$ & 347 & 2 \\
Half-Lives in biota $(\mathrm{d})$ & 16 & 2 \\
Liquid vapor pressure $(\mathrm{Pa})$ & $7.32 \times 10^{-7}$ & 1.5 \\
Water solubility $\left(\mathrm{g} / \mathrm{m}^{3}\right)$ & 0.0043 & 1.5 \\
$\log K_{o w}{ }^{*}$ & $6.04\left(25^{\circ} \mathrm{C}\right)$ & 1.1 \\
$\log K_{o a}{ }^{*}$ & $10.8\left(25^{\circ} \mathrm{C}\right)$ & 1.5 \\
$\log H^{*}(\mathrm{~Pa} \mathrm{~m} / \mathrm{mol})$ & $12.02-3558(\mathrm{~T} / \mathrm{K})$ & 1.5 \\
\hline
\end{tabular}

* : $K_{o w}$ and $K_{o c}$ are octanol-water and water-organic partition coefficient, respectively. $H$ is Henry's law constant. $H$ is temperate-dependent. $C f$ is confidence factor. 


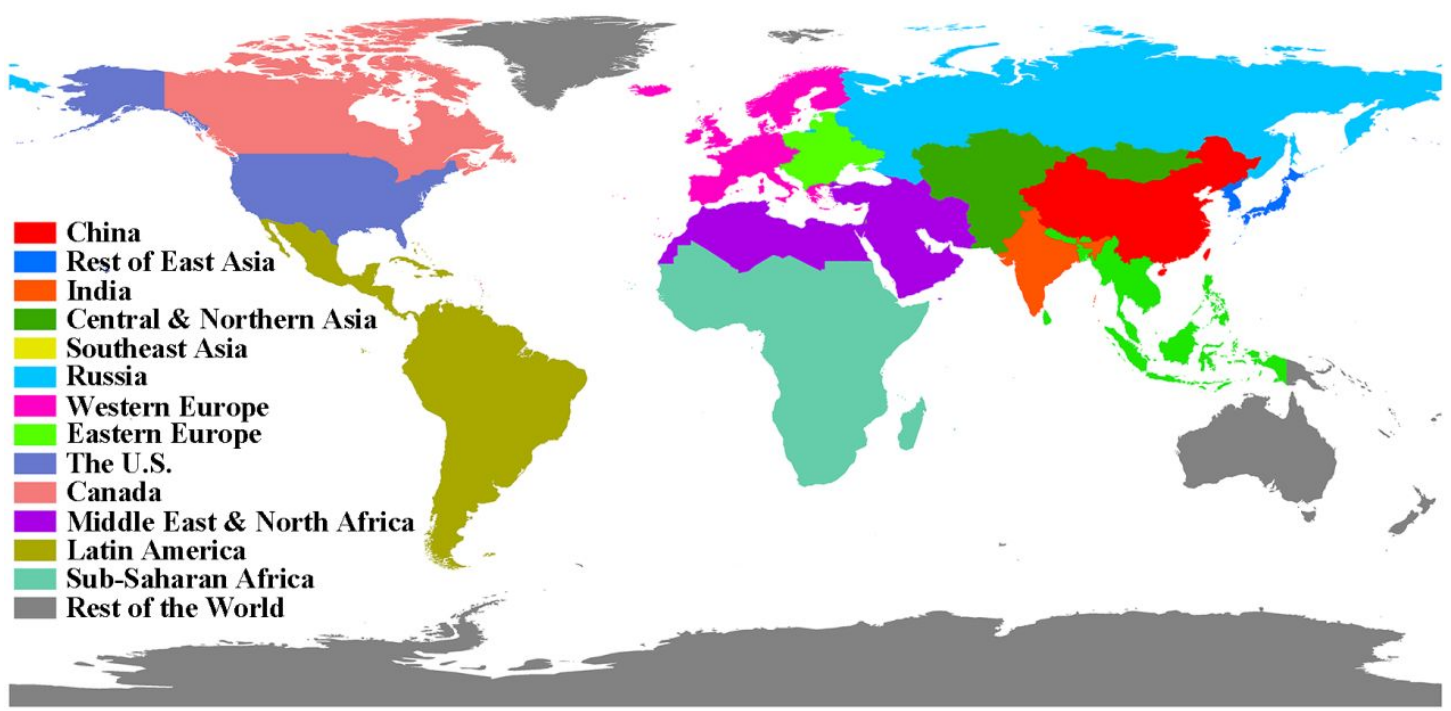

Figure S1. Definition of 14 regions specified in Table S1.
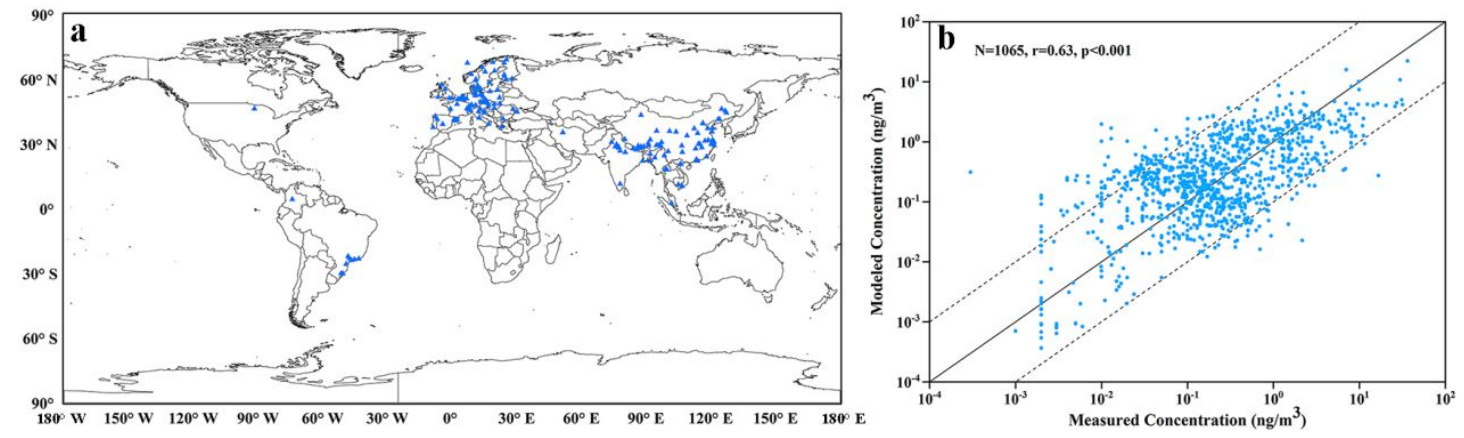

Figure S2. Comparison between modeled and measured BaP air concentrations. (a) Sampling sites assembled from various field campaigns. (b) Scatter plot of modeled and measured BaP air concentrations. The solid black line represents a 1:1 relationship, and the dashed black lines denote the boundaries where modeled concentrations are 0.1 and 10 times of measured concentrations. The data are presented in log-scale. 

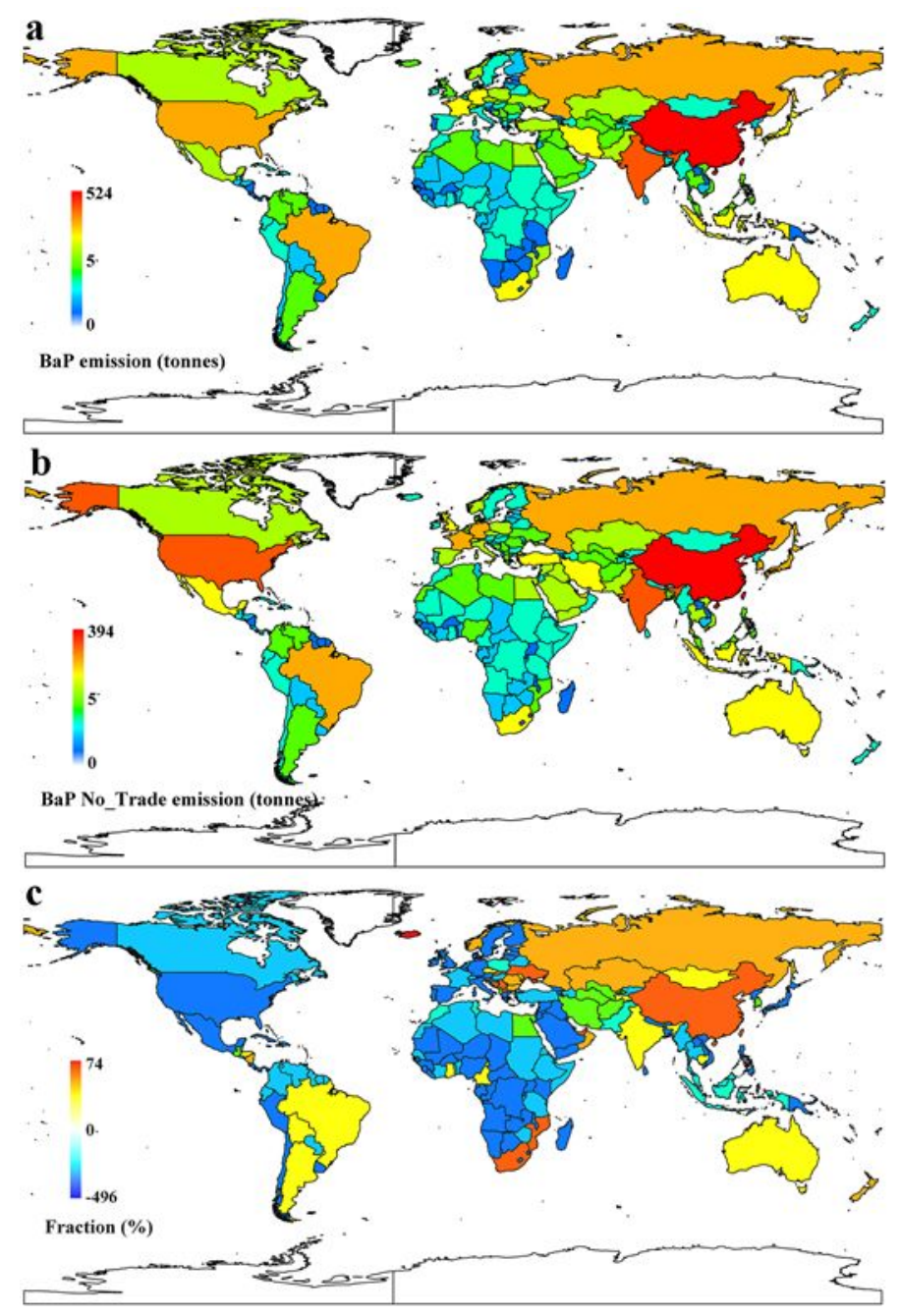

Figure S3. BaP emission (ton/yr) and emission fraction (\%) in 2014. (a) production-based emissions (tonnes, TRADE scenario) in 141 GTAP regions (SI Table S1); (b). BaP emission without taking international trade into account (tonnes, NO_TRADE scenario); (c) fractions of production-based $\mathrm{BaP}$ emission ( $\left.\mathrm{E}_{\mathrm{TRADE}}\right)$ to its $\mathrm{NO}_{-} \mathrm{TRADE}$ emission $\left(\mathrm{E}_{\mathrm{NO}}\right.$ TRADE$)$, defined by $\mathrm{f}=\left(\mathrm{E}_{\mathrm{TRADE}}-\mathrm{E}_{\mathrm{NO} \_\mathrm{TRADE}}\right) / \mathrm{E}_{\mathrm{TRADE}} \times 100(\%)$.

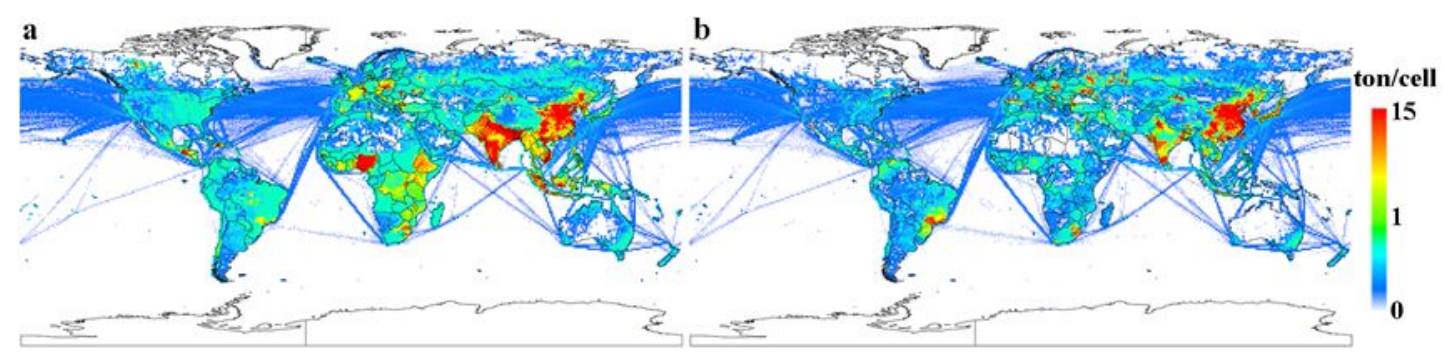

Figure S4. BaP emissions from all sources (a, BASE scenario) and industrial sources (b, TRADE scenario) in 2014, collected from the PKU-FUEL PAH inventory ${ }^{1}$. 


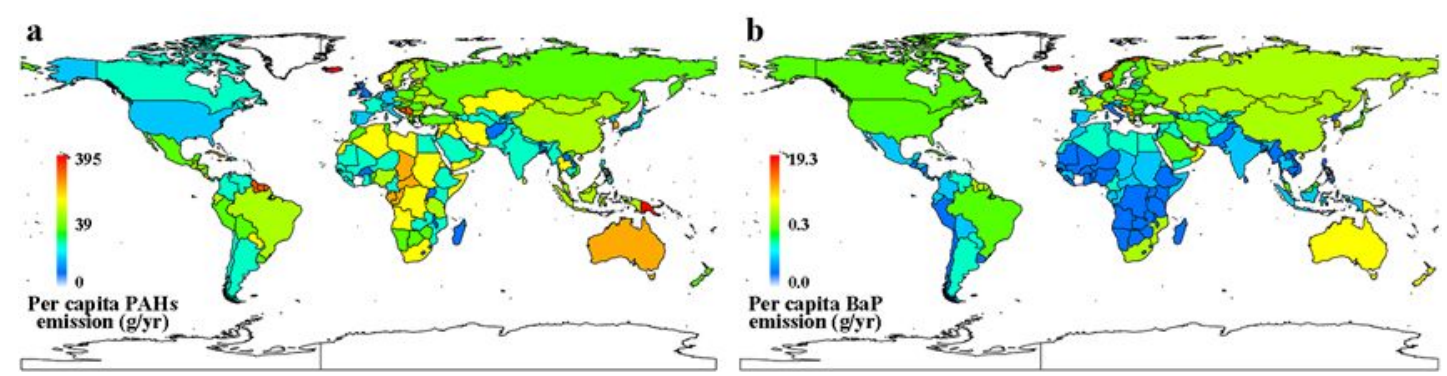

Figure S5. Per capital PAHs (a) and BaP (b) emissions (g/yr) across the global under TRADE scenario in 2014.

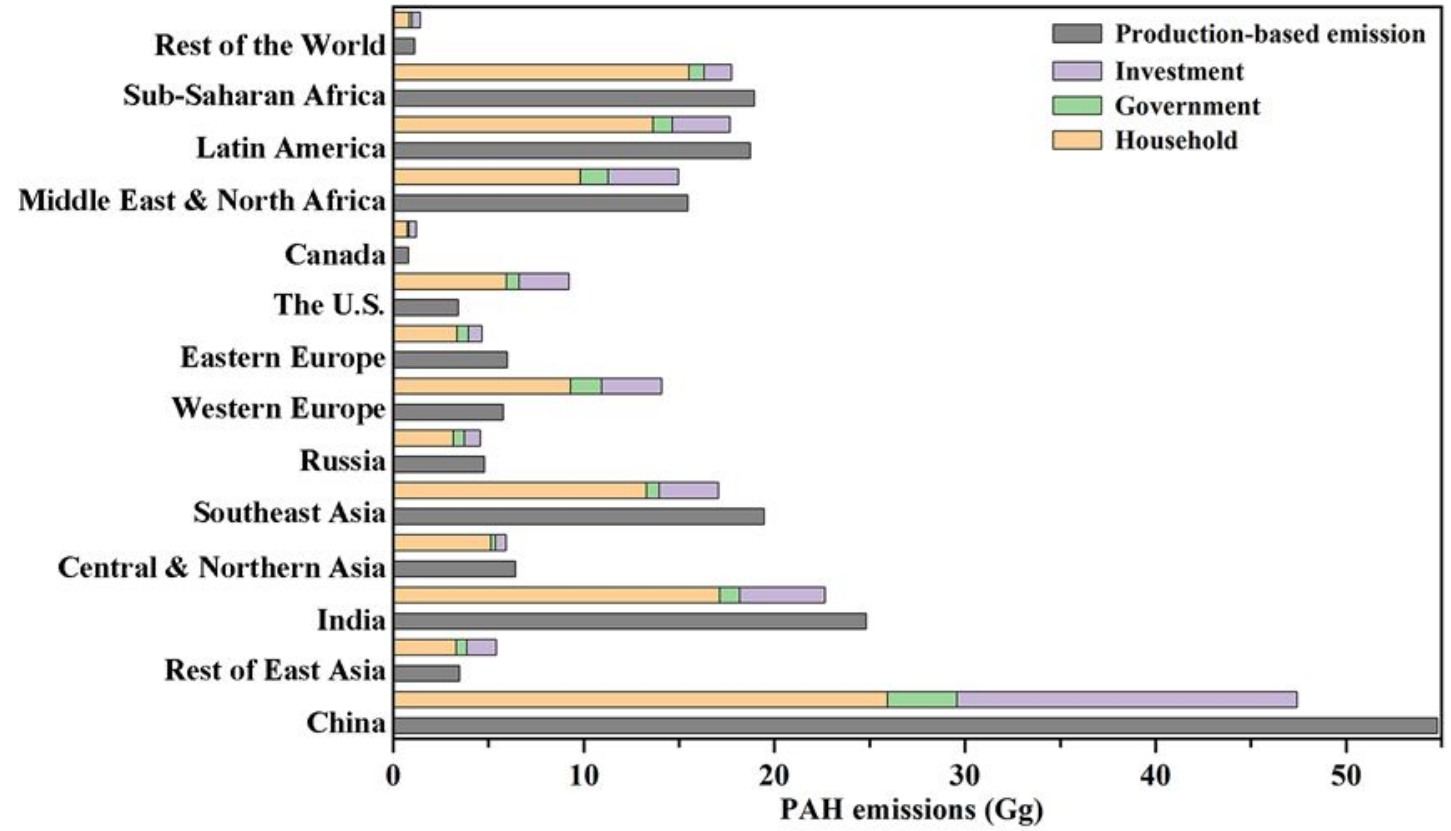

Figure S6. Comparison between PAH production-based and consumption-based emissions (Gg) in 14 regions in 2014. Note: bottom gray bar represents production-based emissions, top bar represents consumption-based emissions (different color represent different type of final consumption). 


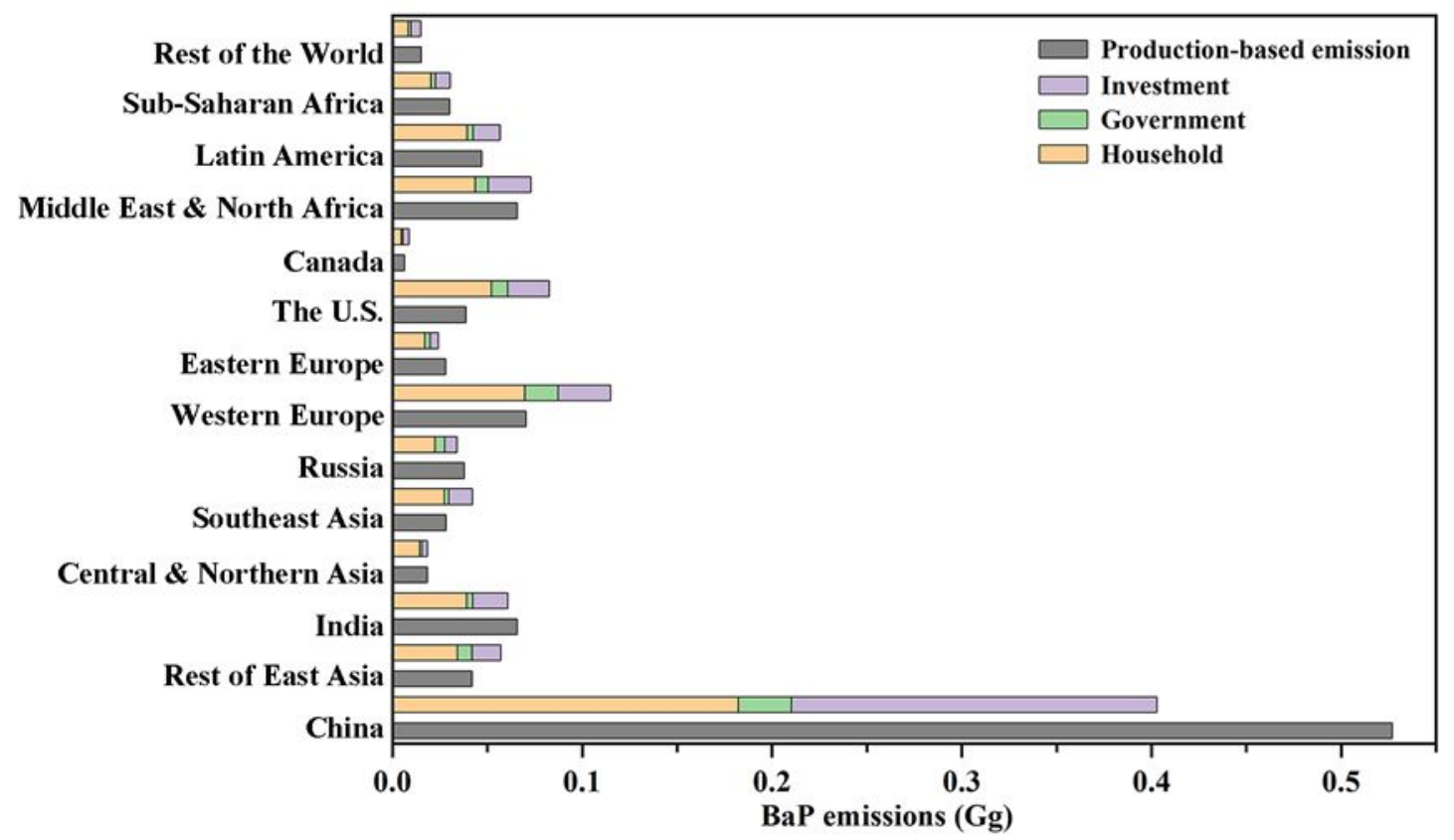

Figure S7. Comparison between BaP production-based and consumption-based emissions ( $\mathrm{Gg})$ in 14 regions in 2014. Note: bottom gray bar represents production-based emissions, top bar represents consumption-based emissions (different color denote different type of final consumption).

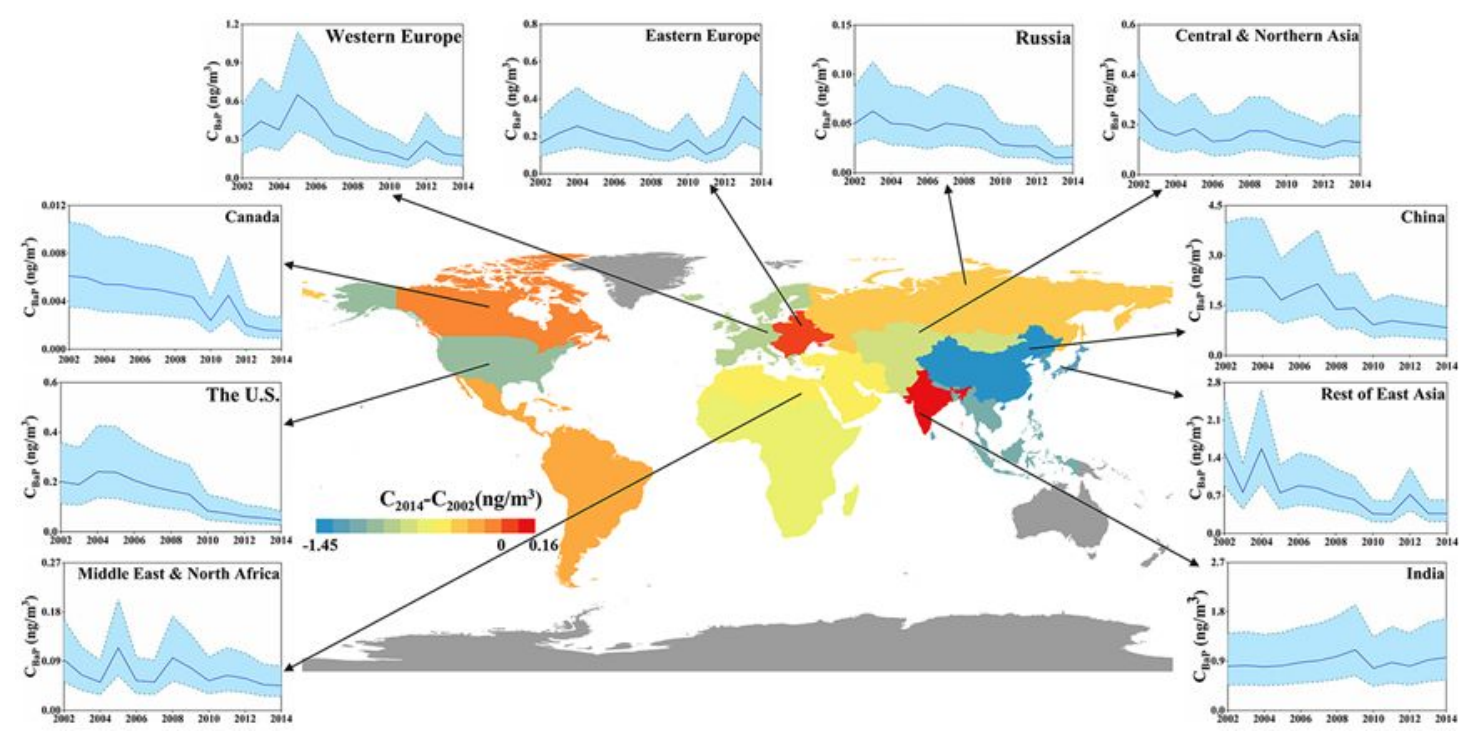

Figure S8. Changes in atmospheric BaP concentration ( $\left.\mathrm{ng} / \mathrm{m}^{3}\right)$ from 2002 to 2014. 


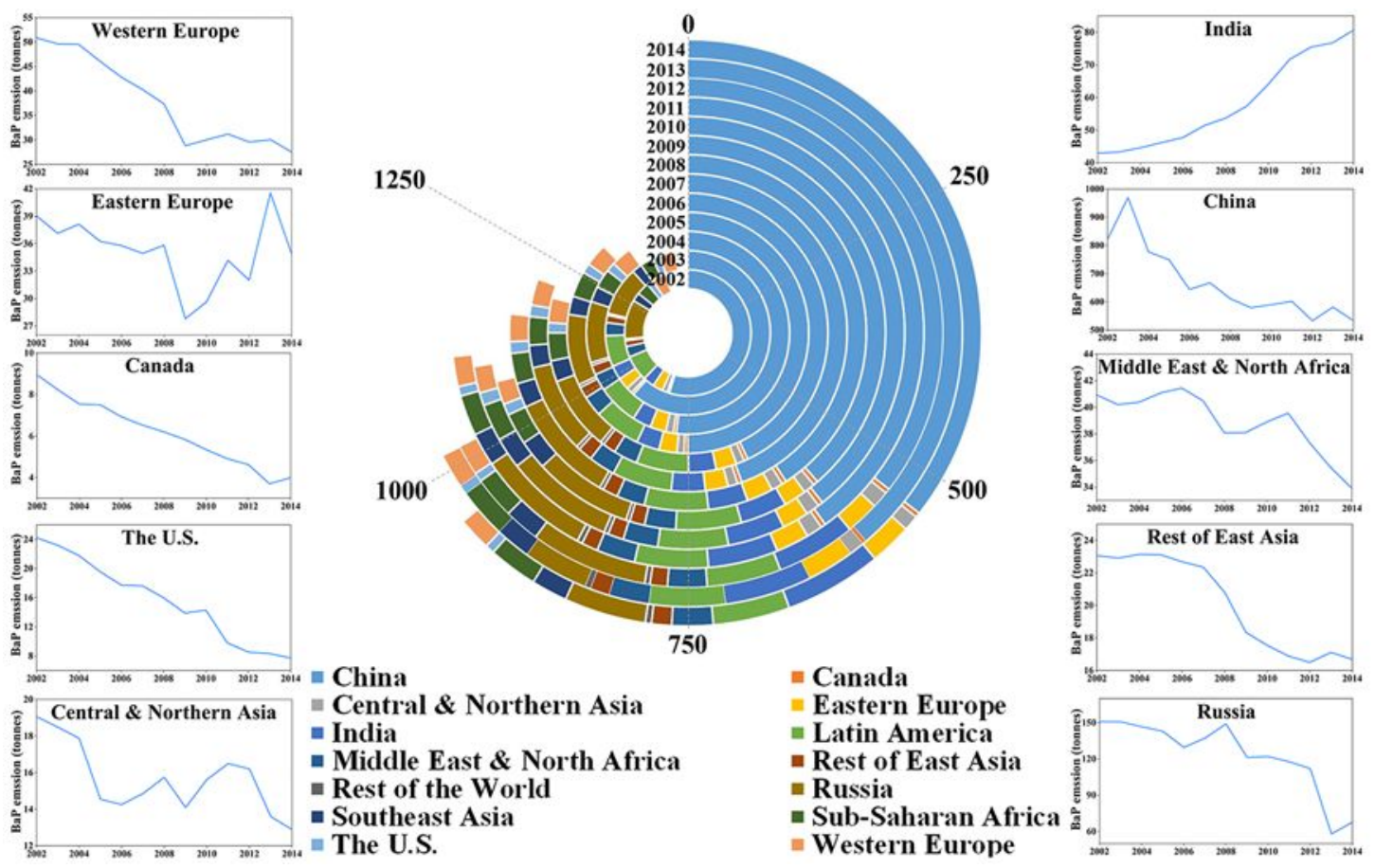

Figure S9. Changes in BaP annual emissions (ton/yr) from 2002 to 2014.
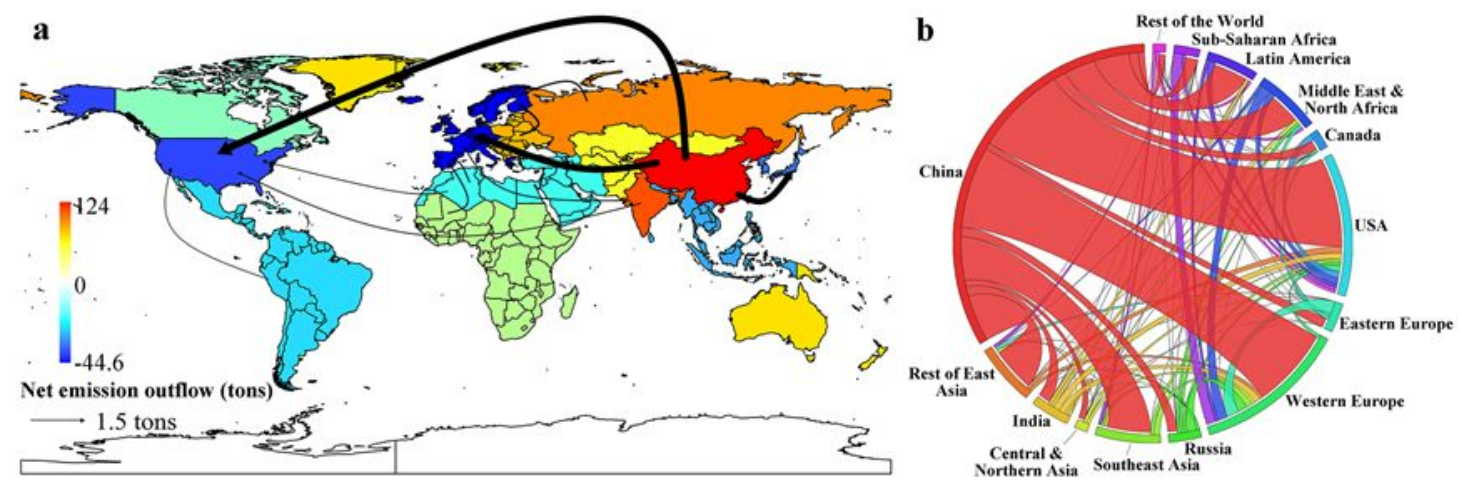

Figure S10. Net international $\mathrm{BaP}$ emission flows (a) and interregional $\mathrm{BaP}$ emission flows (b) within globe in 2014. The 14 outer circular arcs represent 14 regions. Note: the length of each circular arc indicates the magnitude of $\mathrm{BaP}$ emissions transfer in this region, the strips connected with outer circular arcs represent $\mathrm{BaP}$ emissions outflows embodied in this region transfer to other regions and width of the strips represent the magnitude of $\mathrm{BaP}$ emissions transfer. The China had the largest $\mathrm{BaP}$ emission outflows, while Western Europe and the U.S. had the largest BaP emission inflows. 

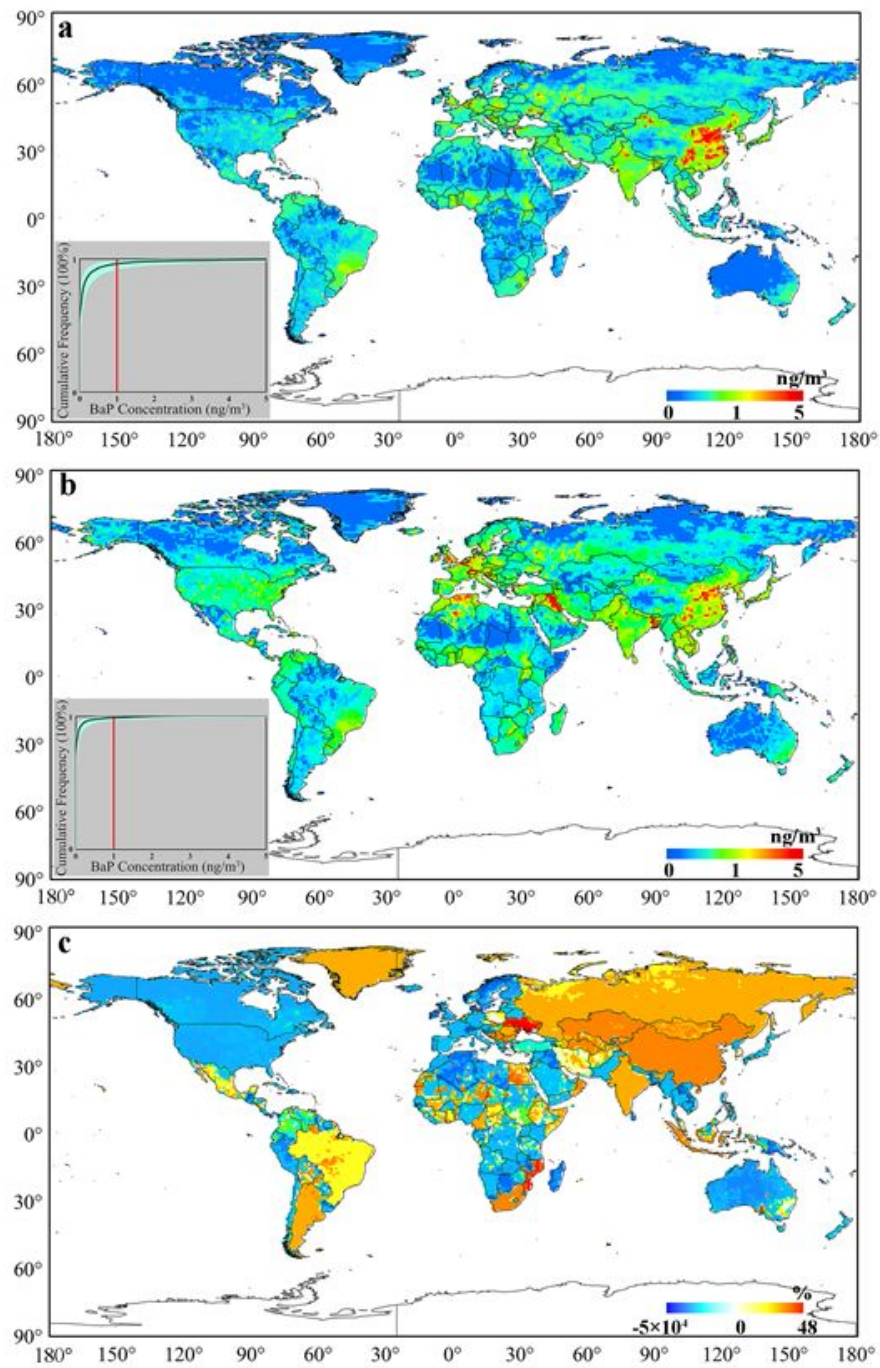

Figure S11. CanMETOP simulated annually averaged BaP concentration across the globe from TRADE run (a. model scenario 1) and NO_TRADE run (b. model scenario 2) in 2014, the fraction of BaP concentration (c) fraction of TRADE (a. model scenario 1) to NO_TRADE run (b. model scenario 2), estimated as $C_{f}=\left[\left(C_{T R A D E}-C_{N O_{-} T R A D E}\right) / C_{T R A D E}\right](100 \%)$. 

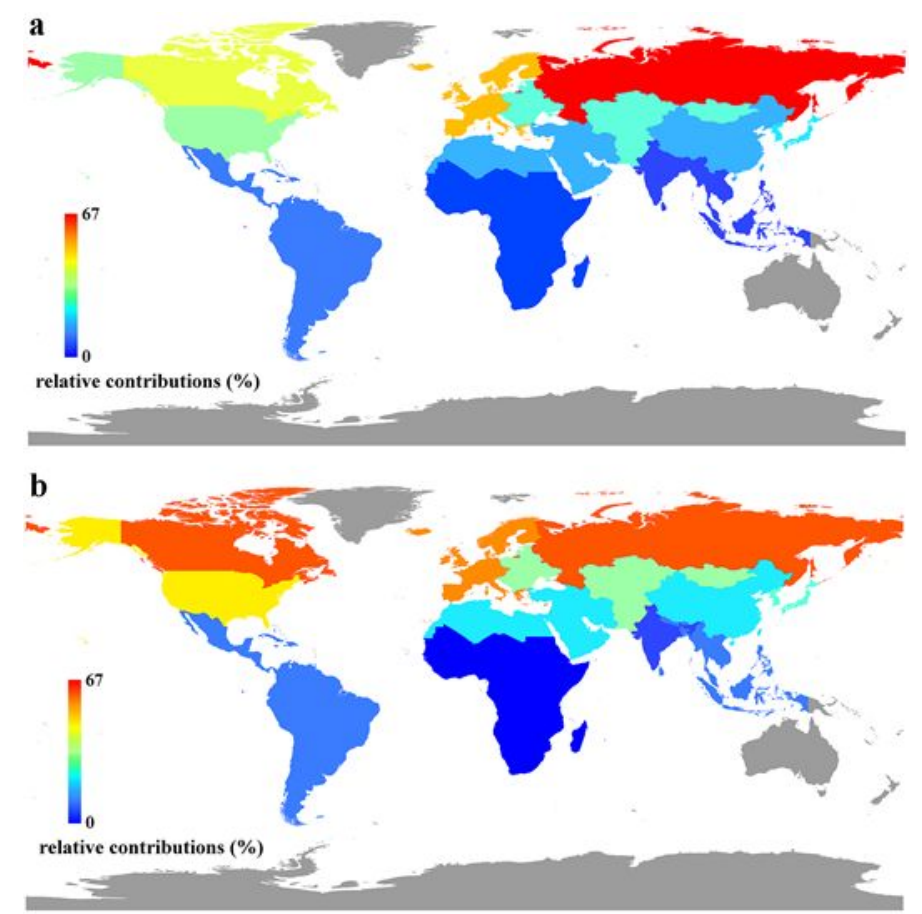

Figure S12. Relative contributions from different regions to the BaP concentration over the Arctic from TRADE scenario (a) and NO_TRADE scenario (b).

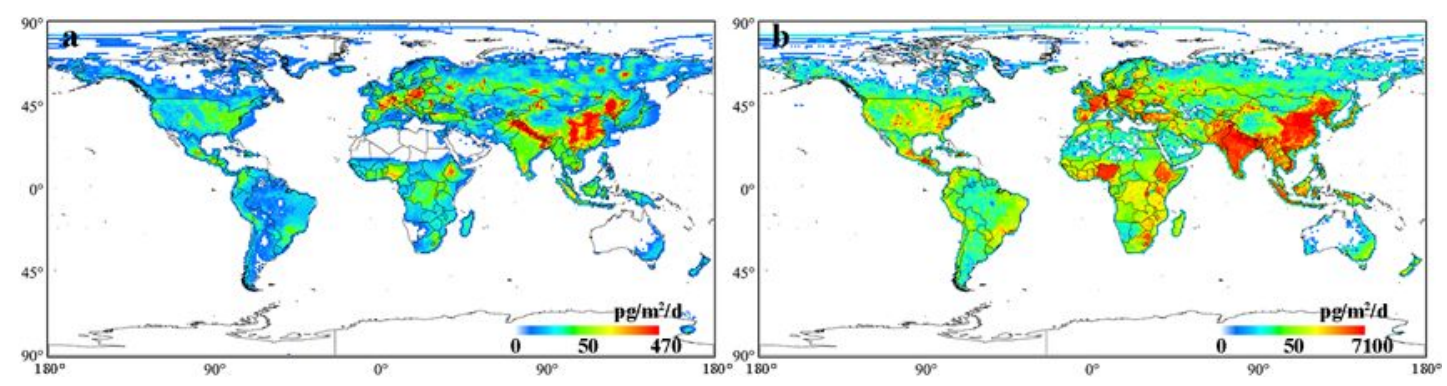

Figure S13. BaP dry (a) and wet (b) deposition fluxes $\left(\mathrm{pg} / \mathrm{m}^{2} / \mathrm{d}\right)$ across the globe under TRADE scenario in 2014. 


\section{References}

1. Shen, H.; Huang, Y.; Wang, R.; Zhu, D.; Li, W.; Shen, G.; Wang, B.; Zhang, Y.; Chen, Y.; Lu, Y.; Chen, H.; Li, T.; Sun, K.; Li, B.; Liu, W.; Liu, J.; Tao, S., Global atmospheric emissions of polycyclic aromatic hydrocarbons from 1960 to 2008 and future predictions. Environ. Sci. Technol. 2013, 47(12), 6415-6424; https://doi.org/10.1021/es400857z.

2. Andrew, R. M.; Peters, G. P., A multi-region input-output table based on the global trade analysis project database (GTAP-MRIO). Econ. Syst. Res. 2013, 25(1), 99-121; https://doi.org/10.1080/09535314.2012.761953.

3. Zhang, Y.; Tao, S.; Shen, H.; Ma, J., Inhalation exposure to ambient polycyclic aromatic hydrocarbons and lung cancer risk of Chinese population. Proc. Natl. Acad. Sci. U. S. A. 2009, 106(50), 21063-21067; https://doi.org/10.1073/pnas.0905756106.

4. Huang, T.; Ling, Z.; Ma, J.; Macdonald, R. W.; Gao, H.; Tao, S.; Tian, C.; Song, S.; Jiang, W.; Chen, L.; Chen, K.; Xie, Z.; Zhao, Y.; Zhao, L.; Gu, C.; Mao, X., Human exposure to polychlorinated biphenyls embodied in global fish trade. Nature Food 2020, 1(5), 292-300; https://doi.org/10.1038/s43016-020-0066-1.

5. Ma, J.; Daggupaty, S.; Harner, T.; Li, Y., Impacts of lindane usage in the Canadian prairies on the Great Lakes ecosystem. 1. Coupled atmospheric transport model and modeled concentrations in air and soil. Environ. Sci. Technol. 2003, 37(17), 3774-3781; https://doi.org/10.1021/es034160x.

6. Ma, J.; Hung, H.; Blanchard, P., How do climate fluctuations affect persistent organic pollutant distribution in North America? Evidence from a decade of air monitoring. Environ. Sci. Technol. 2004, 38(9), 2538-2543; https://doi.org/10.1021/es0349610.

7. Huang, T.; Zhang, X.; Ling, Z.; Zhang, L.; Gao, H.; Tian, C.; Guo, J.; Zhao, Y.; Wang, L.; Ma, J., Impacts of Large-Scale Land-Use Change on the Uptake of Polycyclic Aromatic Hydrocarbons in the Artificial Three Northern Regions Shelter Forest Across Northern China. Environ. Sci. Technol. 2016, 50(23), 12885-12893; https://doi.org/10.1021/acs.est.6b04835. 